\title{
Competência acadêmica de crianças do Ensino Fundamental: características sociodemográficas e relação com habilidades sociais ${ }^{1}$
}

\author{
Marina Bandeira \\ Sandra Silva Rocha \\ Luisa Gonçalves Pires \\ Universidade Federal de São João del Rei \\ Zilda Aparecida Pereira Del Prette \\ Almir Del Prette \\ Universidade Federal de São Carlos
}

\begin{abstract}
RESUMO
Esta pesquisa avaliou características da competência acadêmica em sua relação com o repertório de habilidades sociais e com variáveis sociodemográficas de uma amostra de 257 crianças de $1^{\mathrm{a}}$ a $4^{\mathrm{a}}$ séries do Ensino Fundamental. Foi aplicada a escala SSRS (Social Skills Rating System) a 185 crianças, seus pais e 12 professores. O questionário Critério Brasil foi usado para avaliação sócio-econômica. Os resultados mostraram que $7,9 \%$ dos estudantes apresentavam muita dificuldade de aprendizagem. As meninas obtiveram escores mais elevados do que os meninos em competência acadêmica. As crianças de escola particular apresentaram melhores índices do que as de escolas públicas. Uma melhor competência foi observada em crianças de nível socioeconômico mais elevado. Quanto maior era o nível das habilidades sociais das crianças, melhor era a competência acadêmica e menor o número de reprovações. Estes resultados confirmam os dados da literatura e sugerem a necessidade de intervenções visando promover as habilidades sociais em contexto escolar.
\end{abstract}

Palavras-chave: competência acadêmica; habilidades sociais; crianças; ensino fundamental; variáveis sociodemográficas.

\section{ABSTRACT \\ Academic competence in students of Elementary School: sociodemographic pattern and relation to social skills}

This research investigated academic competence and its relation to social skills and sociodemographic variables in a sample of 257 elementary school students from First through Fourth Grades, using the SSRS scale - Social Skills Rating System. Socioeconomic level was assessed using the "Critério Brasil" scale. Participants were 185 parents and 12 teachers who also rated the children's social skills. Results showed that 7,9\% of the sample had learning difficulties. Girls had higher scores than boys in academic competence. The competence in school was significantly and positively correlated with socioeconomic level. Also, children from private schools had higher scores than those from public schools. There was a significant relationship between social skills and academic competence, the children with higher social skills scores had the better academic competence and were less likely to fail in school. These results confirm previous findings suggesting that social skills interventions in school can affect the students' outcome positively.

Keywords: social skills; academic performance; elementary school students; sociodemographic variables.

Os problemas relacionados à educação escolar, fracasso escolar e dificuldades de aprendizagem são bastante conhecidos no Brasil e alvo de várias discussões. Muitos autores têm apontado para a elevada ocorrência de dificuldades de aprendizagem em estu- dantes de Ensino Fundamental em nosso país (Del Prette \& Del Prette, 1998; 2003; Freller, 1999; Patto, 1987). Crianças com dificuldades escolares geralmente manifestam problemas que afetam seu estado emocional e comportamental, podendo interferir na 
sua vida familiar e nas relações com as demais pessoas (Medeiros \& Loureiro, 2004).

Segundo Erickson (1976), o desenvolvimento socioemocional se processa por etapas, cada qual com suas tarefas e desafios próprios. A resolução satisfatória ou o fracasso em uma dada etapa influencia as etapas posteriores do desenvolvimento. Uma etapa importante para o desempenho acadêmico de crianças ocorre dos 6 aos 12 anos de idade, que corresponde ao início da escolarização. A criança, nesta fase, tem necessidade de aprender com os adultos e de se mostrar competente e com capacidade produtiva, buscando reconhecimento social (Erickson, 1976).

Há períodos da vida, chamados períodos sensíveis por Bronfenbrener (1996), em que determinadas influências são de maior impacto para o desenvolvimento. O processo inicial de escolarização pode ser considerado um período sensível para a trajetória do desempenho escolar (Marturano \& Loureiro, 2003). Segundo Severson e Walker (2002), os dois maiores desafios da socialização, enfrentados pela criança em idade escolar, consistem em se ajustar às demandas do professor e em responder às expectativas dos colegas. Se a criança falhar nesses desafios, pode ter, como conseqüência, o risco do fracasso escolar e da rejeição pelos colegas, como também de desenvolver outros padrões de comportamentos problemáticos (Del Prette \& Del Prette, 2003).

O desempenho escolar constitui uma condição de proteção ou de vulnerabilidade para o desenvolvimento posterior (Marturano \& Loureiro, 2003). O bom desempenho acadêmico favorece a valorização da criança pelos adultos significativos, a superação de dificuldades e o seu engajamento em projetos de vida que busquem a auto-realização. Por outro lado, o baixo desempenho escolar acentua as dificuldades e as vivências de menos valia das crianças, podendo acarretar outras dificuldades comportamentais e emocionais e gerar um senso de não cumprimento de suas tarefas sociais referentes à escolarização (Lindahl, 1998; Marturano, 1997; Medeiros, Loureiro, Linhares \& Marturano, 2000).

Diversos estudos foram realizados visando identificar fatores associados à competência acadêmica, tais como gênero, nível socioeconômico, aspectos cognitivos e desenvolvimento social da criança. Por exemplo, sobre a influência do gênero na competência acadêmica, três estudos (Barbosa, 1999; Carvalho, 2004; Souza, Teixeira e Silva, 2003) concluíram que meninas possuíam melhor desempenho acadêmico que meninos.
A influência do nível socioeconômico sobre o desempenho acadêmico foi avaliada nos estudos de Carvalho (2004), Barbosa (1999) e Ferrão, Beltrão e Santos (2001), tendo sido verificado que os alunos com maior nível socioeconômico possuíam melhor competência acadêmica. No que se refere aos aspectos cognitivos, Dal Vesco, Mattos, Beninca e Ciomara (1998) observaram que alunos de escola particular apresentavam melhor desempenho do que os de escola pública em teste de inteligência, medida pelo WISC. Os alunos de escola particular obtiveram pontuações significativamente melhores em compreensão e vocabulário do que alunos de escola pública.

Ao lado desses fatores sociodemográficos, um corpo crescente de estudos vem focalizando aspectos relacionados ao funcionamento social da criança. Segundo Marturano, Linhares e Parreira (1993), as dificuldades de aprendizagem se caracterizam como uma síndrome psicossocial, ou seja, como um conjunto de fatores que se articulam para produzir o fracasso escolar. Entre tais fatores se incluem as dificuldades nas interações sociais com estudos mostrando que um repertório bem elaborado de habilidades sociais, na idade préescolar, está associado a um maior sucesso escolar posterior e que, ao contrário, um repertório inicial pobre dessas habilidades permite prever dificuldades na adaptação e rendimento escolar (Ferreira \& Marturano, 2002; Marinho, 2003; Del Prette \& Del Prette, 2003).

A competência social qualifica a proficiência do desempenho social e se refere à capacidade do indivíduo de organizar pensamentos, sentimentos e ações em função de seus objetivos e valores, articulando-os às demandas imediatas e mediatas do ambiente (Del Prette \& Del Prette, 2001). Esta capacidade envolve um conjunto de habilidades complexas, incluindo, dentre outras, as de fazer perguntas, lidar com críticas, seguir regras, solicitar mudanças de comportamento e resolver situações interpessoais conflituosas. As habilidades sociais da criança mais freqüentemente enfatizadas e valorizadas nos estudos internacionais dos últimos anos, segundo Del Prette e Del Prette (2003; 2005), podem ser agrupadas em cinco conjuntos de comportamentos: 1. Relação com os companheiros (cumprimentar, elogiar, oferecer ajuda ou assistência, convidar para jogo de interação), 2. Autocontrole (controlar o humor, seguir regras, respeitar limites), 3. Habilidades sociais acadêmicas (envolver-se na tarefa, realizá-la de forma independente, seguir instruções), 4. Ajustamento (seguir regras e comportar-se de acordo com o esperado), e 5. Asserção (iniciar conversação, aceitar elogios, fazer convites). 
As habilidades sociais possuem um caráter situacional, ou seja, variam conforme o contexto social e a cultura. Por exemplo, Bandeira, Freitas, Del Prette e Del Prette (no prelo) observaram escores de habilidades sociais mais elevados em crianças de escola particular, de nível socioeconômico e escolaridade mais elevados dos pais, do sexo feminino e de menor idade.

As habilidades sociais são adquiridas por meio do processo de aprendizagem. Desenvolvem-se desde a infância e este é considerado como um período crítico para aprendizagem dessas habilidades, o que coloca em destaque a necessidade de focalizar a atenção para o desenvolvimento das habilidades sociais de crianças (Caballo, 2003; Del Prette \& Del Prette, 1999).

A associação entre competência social e acadêmica tem sido objeto de muitos estudos, parte deles avaliando especificamente as habilidades sociais e outros avaliando indicadores indiretos desse repertório, como a qualidade das relações da criança com seus pares e/ou com adultos (Del Prette \& Del Prette, 2002; 2003; Marturano e cols., 1993). Alguns estudos são descritivos das crianças com dificuldades de aprendizagem e outros comparam crianças com bom rendimento acadêmico e crianças com baixo rendimento ou mesmo com dificuldades de aprendizagem (Estevanato, Loureiro, Linhares \& Marturano, 2003; Lemos \& Menezes, 2002; Marturano e cols., 1993; Medeiros e cols., 2000; Medeiros, Loureiro, Linhares \& Marturano, 2003).

Sobre as relações entre indicadores diretos ou indiretos de habilidades sociais e a competência acadêmica, a pesquisa de Marturano e cols. (1993), analisando apenas um grupo de crianças com queixa de dificuldade de aprendizagem, constatou que elas apresentavam concomitantemente problemas nas suas interações sociais. No estudo de Lemos e Meneses (2002), comparando crianças com competência acadêmica alta e baixa, verificou-se que o primeiro grupo possuía um repertório social mais bem elaborado do que o segundo, avaliado por meio do Sistema de Avaliação de Habilidades Sociais (SSRS), de Gresham e Elliott (1990).

Pesquisas avaliando a relação entre competência acadêmica e habilidades sociais, por meio da comparação de grupos de crianças, com e sem dificuldades de aprendizagem (Estevanato e cols., 2003; Medeiros e cols., 2003), mostraram que alunos com dificuldades de aprendizagem apresentavam maiores déficits em interações sociais. Estes dados são corroborados por dois estudos de Del Prette e Del Prette (2002, 2003), com base na avaliação das habilidades sociais e da competência social por meio, respectivamente, de um instrumento multimídia e de protocolos respondidos pela própria criança, seus pares e professores.

Os resultados das investigações de comparação de grupos (Estevanato e cols., 2003; Lemos \& Medeiros, 2002; Medeiros e cols., 2003) sugerem que a promoção da competência social das crianças pode favorecer o desempenho acadêmico. Essa hipótese foi testada mais diretamente em um estudo, no qual se promoveu o desenvolvimento do repertório de habilidades sociais, por meio de um programa de Treinamento em Habilidades Sociais, avaliando-se a competência acadêmica e social das crianças no pré-teste e no pós-teste (Molina, 2003). Os resultados mostraram que a promoção do repertório social teve como efeito um aumento do desempenho acadêmico.

Estudos sobre as relações entre estas variáveis são particularmente necessários e relevantes em nosso país, devido à gravidade do fracasso escolar no Brasil (Del Prette \& Del Prette, 1998; 2003) e à necessidade do uso de medidas para a prevenção e redução das dificuldades de aprendizagem. Por exemplo, o estudo realizado por Carneiro, Martinelli e Sisto (2003), avaliando o desempenho na escrita de crianças de escolas públicas da $3^{\mathrm{a}}$ série de Ensino Fundamental de Campinas, mostrou que 15,2\% da amostra apresentavam dificuldades neste desempenho. Estas dificuldades podem variar dependendo da região do país.

Em estudo relativamente recente, Carvalho (2003) mostrou que as dificuldades de aprendizagem eram mais críticas nas regiões Norte e Nordeste. O repertório de habilidades sociais, por seu caráter situacionalcultural, também varia de acordo com características culturais dos grupos sociais (Del Prette \& Del Prette, 1999). Assim, a compreensão acerca da relação entre as habilidades sociais e a competência acadêmica precisa ser contextualizada, o que justifica estudos mais focais e em diferentes contextos regionais.

Dadas as considerações anteriores, a presente pesquisa teve como objetivos: a) identificar a freqüência de ocorrência das dificuldades de aprendizagem em uma amostra de estudantes de uma cidade do interior de Minas Gerais; b) analisar as características da competência acadêmica dessas crianças, em sua relação com variáveis sociodemográficas (sexo, série, tipo de escola e nível socioeconômico); c) relacionar indicadores de competência acadêmica e de dificuldades de aprendizagem com escores de habilidades sociais, obtidos em avaliações junto às crianças, seus pais e seus professores. 


\section{MÉTODO}

\section{Participantes}

Participaram desta pesquisa 257 crianças de $1^{\mathrm{a}}$ a $4^{\mathrm{a}}$ série do ensino fundamental, de duas escolas públicas e uma escola particular de uma cidade do interior de Minas Gerais. Em cada escola, participou uma turma de cada série, totalizando doze turmas. Participaram ainda 185 pais dessas crianças e 12 professores.

As crianças possuíam idade média de 8,62 anos (d.p.=1,49), sendo $111(43,2 \%)$ meninas e $146(56,8 \%)$ meninos. A amostra da escola pública foi composta por 182 crianças $(70,80 \%)$ e a da escola particular por 75 crianças $(29,20 \%)$.

Os pais possuíam idade média de 38,09 anos (d.p.=8,93), sendo que $185(72 \%)$ aceitaram participar da pesquisa e 99 (38\%) se recusaram, mas autorizaram a inclusão dos filhos na pesquisa. Os professores que participaram eram todos do sexo feminino, com idade média de 43,16 anos (d.p.=5,06) e lecionavam para as crianças participantes.

\section{Instrumentos de Medida}

Foram utilizados dois instrumentos de medida: O Social Skills Rating System (SSRS), para avaliar o repertório de habilidades sociais, problemas de comportamento e competência acadêmica das crianças e o Critério de Classificação Econômica Brasil, também conhecido como Critério Brasil, para medir o poder aquisitivo dos participantes.

\section{Sistema de Avaliação de Habilidades Sociais (Social Skills Rating System ou SSRS)}

O SSRS é um sistema de avaliação de habilidades sociais, problemas de comportamento e competência acadêmica de crianças, desenvolvido e validado nos Estados Unidos por Gresham e Elliott (1990). O SSRS é apresentado em três versões: a de auto-avaliação pela criança, a de avaliação pelos pais ou responsáveis e a de avaliação pelo professor. Esse instrumento foi traduzido e adaptado para o Brasil como parte de um projeto temático mais amplo, do qual participam os autores deste trabalho (Del Prette, 2003). Para a amostra deste estudo, foram obtidos índices adequados de consistência interna das escalas, calculada por meio do coeficiente alfa de Cronbach: para as escalas de habilidades sociais (estudantes $=0,77$; pais $=0,86$; professores $=0,92$ ), as duas escalas de avaliação dos comportamentos problemáticos (pais $=0,82$; professores $=0,88)$ e a escala de competência acadêmica (alfa $=0,98)$. Foi utilizado o critério mínimo de correlação item-total superior a 0,20 para retenção dos itens nas escalas.

As três escalas do SSRS incluem a avaliação da frequiência de emissão de habilidades sociais específicas, contendo 35 itens na de auto-avaliação, 38 itens na de avaliação por pais e 30 na de avaliação por professores. Pais e professores respondem também sobre a importância de cada item das habilidades sociais para o desenvolvimento sadio na infância e sobre a frequiência de ocorrência de comportamentos problemáticos da criança (neste caso, 17 e 18 itens, respectivamente).

Para a avaliação da frequiência de habilidades sociais e de comportamentos problemáticos são utilizadas três alternativas de resposta, "nunca" (0), "algumas vezes" (1) e "muito freqüente" (2). Para a avaliação da importância das habilidades sociais também são utilizadas três alternativas: "não importante" (0), "importante" (1) e "muito importante"(2).

O professor avalia ainda a competência acadêmica dos estudantes, em uma escala contendo nove itens, para os quais ele classifica a criança em relação aos demais colegas, através de cinco alternativas de resposta: "entre os $10 \%$ piores" (1); entre os $20 \%$ piores (2); "entre os $40 \%$ médios" (3); "entre os $20 \%$ bons" (4); e "entre os $10 \%$ melhores" (5). Os itens desta escala se referem ao desempenho acadêmico geral, funcionamento intelectual do estudante, nível de motivação geral para o êxito acadêmico, nível de estímulo dos pais para o êxito acadêmico do estudante, desempenho atual em leitura e em matemática e nível destas duas ultimas habilidades em termos da expectativa para a série em que o estudante se encontra.

Além das questões das escalas propriamente ditas, descritas acima, o SSRS inclui ainda duas outras avaliações, que são feitas antes dos respondentes preencherem as questões das escalas. Refere-se a uma classificação geral dos pais e professores sobre a presença ou não de comportamentos problemáticos e de dificuldades de aprendizagem, em cada criança avaliada, segundo uma escala de 1 a 3 , correspondendo a "Não tem", "Pouco" e "Muito".

\section{Critério de Classificação Econômica Brasil (Critério Brasil)}

Este instrumento avalia o nível socioeconômico das famílias, com base em itens referentes ao poder aquisitivo, posse de bens de consumo duráveis e grau de instrução do chefe da família (ABEP, 2003). Estes critérios para classificação econômica no Brasil foram 
estabelecidos pela Associação Brasileira de Anunciantes (ABA), pela Associação Nacional das Empresas de Pesquisa de Mercado (ANEP) e pela Associação Brasileira dos Institutos de Pesquisa de Mercado (ABIPEME), baseados nos levantamentos socioeconômicos de 1993 e 1997.

\section{Procedimento}

Inicialmente, foram contatadas as três escolas participantes. Após esse contato, escolheram-se quatro turmas em cada escola e foram enviadas cartas aos pais destes estudantes, solicitando sua autorização para a participação dos seus filhos na pesquisa.

Em seguida, foi feita a aplicação dos questionários dos estudantes, realizada em sala de aula, de forma coletiva e acompanhada da supervisão dos aplicadores. Os sujeitos que tiveram dificuldades em responder receberam uma supervisão individual.

Para a aplicação do questionário dos pais, foi enviada uma carta marcando uma reunião na escola, na qual eles respondiam aos dois questionários, o SSRS e o Critério Brasil. A aplicação foi coletiva, mas com a supervisão individual do aplicador, quando necessário. Aqueles pais que não compareceram à reunião, foram novamente contatados para que pudessem responder ao questionário, em novo dia e horário.

Para a aplicação dos questionários das professoras, uma reunião foi marcada em horário de trabalho, mediante a autorização da diretora, tendo sido adotado o mesmo procedimento seguido com os pais. As professoras responderam um questionário para cada aluno participante. Ao final das aplicações foi verificado se não havia questões em branco.

\section{RESULTADOS}

A seguir são apresentados os resultados relativos à análise descritiva (em termos percentuais) dos indicadores de competência acadêmica e de dificuldades de aprendizagem na amostra geral. Em seguida, são descritos os resultados das relações entre os escores de competência acadêmica e as variáveis estudadas.

\section{Dificuldade de Aprendizagem}

Os dados mostraram que $7,82 \%$ das crianças da amostra foram classificadas pelos professores como estudantes que apresentavam muita dificuldade de aprendizagem, 23,04\% apresentavam pouca dificuldade de aprendizagem e $69,14 \%$ das crianças não apresentavam nenhuma dificuldade de aprendizagem.

\section{Competência Acadêmica}

A Tabela 1 apresenta as percentagens (\%) de alunos que foram classificados pelas professoras em cada um dos nove itens da escala de avaliação da competência acadêmica.

\begin{tabular}{|c|c|c|c|c|c|c|c|c|c|c|}
\hline \multirow{4}{*}{ Itens } & \multicolumn{10}{|c|}{ Competência acadêmica } \\
\hline & \multirow{2}{*}{\multicolumn{2}{|c|}{$\begin{array}{c}10 \% \\
\text { piores }\end{array}$}} & \multirow{2}{*}{\multicolumn{2}{|c|}{$\begin{array}{l}20 \% \\
\text { piores }\end{array}$}} & \multirow{2}{*}{\multicolumn{2}{|c|}{$\begin{array}{c}40 \% \\
\text { médios }\end{array}$}} & \multirow{2}{*}{\multicolumn{2}{|c|}{$\begin{array}{l}20 \% \\
\text { bons }\end{array}$}} & \multirow{2}{*}{\multicolumn{2}{|c|}{$\begin{array}{c}10 \% \\
\text { ótimos }\end{array}$}} \\
\hline & & & & & & & & & & \\
\hline & $\mathrm{N}$ & $\%$ & $\mathrm{~N}$ & $\%$ & $\mathrm{~N}$ & $\%$ & $\mathrm{~N}$ & $\%$ & $\mathrm{~N}$ & $\%$ \\
\hline Desempenho acadêmico geral & 12 & 4,69 & 17 & 6,64 & 65 & 25,39 & 68 & 26,56 & 94 & 36,72 \\
\hline Leitura & 12 & 4,69 & 17 & 6,64 & 60 & 23,43 & 76 & 29,69 & 91 & 35,55 \\
\hline Matemática & 13 & 5,08 & 15 & 5,86 & 64 & 25,00 & 60 & 23,44 & 104 & 40,62 \\
\hline Expectativa de leitura & 13 & 5,09 & 17 & 6,64 & 57 & 22,26 & 65 & 25,39 & 104 & 40,62 \\
\hline Expectativa de matemática & 11 & 4,30 & 17 & 6,64 & 64 & 25,00 & 58 & 22,66 & 106 & 41,40 \\
\hline Motivação Geral & 11 & 4,30 & 17 & 6,64 & 51 & 19,92 & 77 & 30,08 & 100 & 39,06 \\
\hline Estímulo dos pais & 10 & 3,91 & 16 & 6,25 & 50 & 19,53 & 76 & 29,69 & 104 & 40,62 \\
\hline Funcionamento intelectual & 12 & 4,69 & 18 & 7,03 & 58 & 22,66 & 75 & 29,30 & 93 & 36,32 \\
\hline Comport. geral em classe & 12 & 4,69 & 14 & 5,47 & 52 & 20,31 & 86 & 33,59 & 92 & 35,94 \\
\hline
\end{tabular}

Pode-se observar, na Tabela 1, que em todos os itens que avaliam a competência acadêmica, a maioria dos alunos (entre $35,55 \%$ a $41,40 \%$ da amostra) foi classificada pelo professor entre os "10\% ótimos" e uma minoria de alunos (de 3,91\% a 5,09\% da amostra) entre os " $10 \%$ piores".
Competência Acadêmica por série: Utilizou-se a análise de variância ANOVA para verificar se havia diferenças significativas na competência acadêmica das crianças das quatro séries do ensino fundamental e o teste post hoc Tukey para identificar quais séries apresentavam maior competência acadêmica. Verifi- 
cou-se que as quatro séries não diferiram entre si no escore global de competência acadêmica, pois não houve uma diferença estatisticamente significativa entre elas $(\mathrm{F}[3,256]=0,75 ; p=0,52)$.

Competência Acadêmica por Tipo de Escola: Foi utilizada a análise estatística "t" de Student para verificar se havia diferenças significativas na competência acadêmica por tipo de escola. Verificou-se que os alunos da escola particular obtiveram um escore global de competência acadêmica significativamente mais elevado do que os alunos da escola pública $(\mathrm{t}=$ 4,$24 ; p=0,00$ ).

Competência Acadêmica em Função do Nível Socioeconômico: Uma análise de correlação de Pearson foi usada para verificar a relação entre competência acadêmica e o nível socioeconômico. Foram obtidas correlações positivas significativas entre o escore global de competência acadêmica dos alunos e o nível socioeconômico de suas famílias, pelo Critério Brasil $(\mathrm{r}=0,36 ; p=0,00)$, assim como o grau de escolaridade dos pais $(\mathrm{r}=0,38 ; p=0,00)$. Quanto maior a escolaridade e o nível socioeconômico dos pais, maior era competência acadêmica dos alunos.

Competência Acadêmica em Função do Sexo: Foi utilizada a análise estatística " $t$ " de Student para verificar se havia diferenças significativas na competência acadêmica por sexo. Observou-se uma diferença estatisticamente significativa entre meninos e meninas, quanto ao escore global de competência acadêmica, as meninas se mostraram mais competentes do que os meninos $(\mathrm{t}=-2,06 ; p=0,04)$.

\section{Relação entre Habilidades Sociais e Desempenho na Escola}

A seguir, serão apresentados os dados sobre as relações entre habilidades sociais e o desempenho na escola. Uma análise de correlação de Pearson foi usa- da para verificar a relação entre competência acadêmica e as variáveis referentes a habilidades sociais e número de reprovações.

Habilidades Sociais e Competência Acadêmica: Os resultados mostraram uma correlação positiva significativa entre o escore global de competência acadêmica e os escores de habilidades sociais, nas três avaliações realizadas, dos alunos $(\mathrm{r}=0,23 ; p=0,00)$, dos pais $(\mathrm{r}=0,36 ; p=0,00)$ e dos professores $(\mathrm{r}=0,72$; $p=0,00$ ). Quanto maior o escore global de habilidades sociais das crianças, maior foi a sua competência acadêmica.

Habilidades Sociais e Número de Reprovações: Verificou-se uma correlação negativa significativa entre o número de reprovações das crianças e seus escores de habilidades sociais na avaliação dos alunos $(\mathrm{r}=-0,14 ; p=0,02)$ e das professoras $(\mathrm{r}=-0,16 ; p=0,01)$. Assim, quanto maior o escore global de habilidades sociais das crianças, menor foi seu número de reprovações. Entretanto, na avaliação das habilidades sociais realizada pelos pais, não se observou uma correlação entre essas duas variáveis $(\mathrm{r}=-0,07 ; p=0,32)$.

\section{Habilidades Sociais em Subgrupos com Melhor e Pior Competência Acadêmica}

Serão apresentados, a seguir, os dados de comparação das habilidades sociais de dois grupos de alunos, os classificados pelas professoras na categoria " $10 \%$ piores" da classe e os classificados entre os "10\% ótimos", para itens específicos da escala de competência acadêmica. Foi utilizada a análise estatística " $t$ " de Student para verificar se havia diferenças significativas entre os grupos.

Desempenho Acadêmico Geral: A Tabela 2 mostra as médias dos escores globais de habilidades sociais dos alunos com pior e melhor desempenho acadêmico geral.

Tabela 2: Médias, desvios-padrão $(d p)$, valores de t de Student e nível de significância $(p)$ das habilidades sociais dos grupos dos piores e dos melhores alunos no desempenho acadêmico geral

\begin{tabular}{lllllll}
\hline \multirow{2}{*}{ Escalas } & \multirow{2}{*}{ Grupos } & $\mathbf{N}$ & Média & $\mathbf{d p}$ & $\mathbf{t}$ & $\boldsymbol{p}$ \\
\cline { 2 - 6 } & & 12 & 44,42 & 7,46 & $-2,90^{\star}$ & \multirow{2}{*}{0,00} \\
\hline \multirow{2}{*}{ Estudantes } & Piores & 94 & 50,47 & 6,70 & & \\
& Melhores & 10 & 35,70 & 9,96 & $-4,71^{\star}$ & 0,00 \\
\multirow{2}{*}{ Pais } & Piores & 64 & 50,59 & 9,18 & & \\
\multirow{2}{*}{ Professoras } & Melhores & 12 & 23,92 & 6,61 & $-12,97^{\star}$ & 0,00 \\
& Piores & 94 & 48,78 & 6,21 & & \\
\hline
\end{tabular}


Observa-se, na Tabela 2, que os alunos com melhor desempenho acadêmico geral apresentaram escores significativamente mais elevados de habilidades sociais do que os alunos com pior desempenho acadêmico geral. Este resultado foi o mesmo quando as habilidades sociais foram avaliadas pelos estudantes $(\mathrm{t}=-2,90 ; p=0,00)$, pelos pais $(\mathrm{t}=-4,71 ; p=0,00) \mathrm{e}$ pelos professores $(\mathrm{t}=-12,97 ; p=0,00)$.
Desempenho em Leitura: A Tabela 3 mostra os escores de habilidades sociais obtidos pelos grupos dos piores e dos melhores alunos em leitura. Pode-se observar, na Tabela 3, que os melhores alunos em leitura apresentaram escores significativamente mais elevados de habilidades sociais do que os piores em leitura. Este resultado foi o mesmo quando as habilidades sociais foram avaliadas pelos estudantes $(\mathrm{t}=$ $2,14 ; p=0,03)$, pelos pais $(\mathrm{t}=-5,43 ; p=0,00)$ e pelos professores $(\mathrm{t}=-11,16 ; p=0,00)$.

Tabela 3: Médias, desvios-padrão $(d p)$ e valores de $t$ de Student das habilidades sociais dos grupos dos piores e dos melhores alunos em matemática e leitura

\begin{tabular}{|c|c|c|c|c|c|c|c|c|c|}
\hline \multirow{2}{*}{ Escalas } & \multirow{2}{*}{ Grupos } & \multicolumn{4}{|c|}{ Matemática } & \multicolumn{4}{|c|}{ Leitura } \\
\hline & & $\mathrm{N}$ & Média & $d p$ & $t$ & $\mathrm{~N}$ & Média & $d p$ & $t$ \\
\hline \multirow{2}{*}{ Estudantes } & Piores & 13 & 44,5 & 7,16 & \multirow{2}{*}{$-2,75^{\star \star}$} & 12 & 45,25 & 7,11 & \multirow{2}{*}{$-2,14^{*}$} \\
\hline & Melhores & 104 & 50,30 & 7,11 & & 91 & 50,12 & 7,44 & \\
\hline \multirow{2}{*}{ Pais } & Piores & 10 & 35,70 & 9,97 & \multirow{2}{*}{$-5,07^{\star \star}$} & 9 & 34,67 & 9,81 & \multirow{2}{*}{$-5,43^{\star *}$} \\
\hline & Melhores & 75 & 50,97 & 8,81 & & 63 & 51,06 & 8,28 & \\
\hline \multirow{2}{*}{ Professores } & Piores & 13 & 25,15 & 7,74 & \multirow{2}{*}{$-11,79^{* *}$} & 12 & 25,67 & 7,35 & \multirow{2}{*}{$-11,16^{\star *}$} \\
\hline & Melhores & 104 & 47,98 & 6,43 & & 91 & 48,17 & 6,46 & \\
\hline
\end{tabular}

Desempenho em Matemática: A Tabela 3 mostra que os melhores alunos em matemática apresentaram escores significativamente mais elevados em habilidades sociais do que os piores alunos em matemática. Este resultado foi o mesmo quando as habilidades sociais foram avaliadas pelos estudantes $(t=-2,75$; $p=0,00)$, pelos pais $(\mathrm{t}=-5,07 ; p=0,00)$ e pelos professores $(\mathrm{t}=-11,79 ; p=0,00)$.

Estímulo dos Pais para o Êxito Acadêmico: A Tabela 4 mostra os escores de habilidades sociais obtidos pelos alunos que possuíam maior e menor estímulo dos pais para o êxito acadêmico.

Tabela 4: Médias, desvios-padrão $(d p)$ e valores de t de Student das habilidades sociais dos grupos de alunos com maior e menor estímulo dos pais para o êxito acadêmico, motivação geral e funcionamento intelectual

\begin{tabular}{|c|c|c|c|c|c|c|c|c|c|c|}
\hline \multirow{2}{*}{ Escalas } & \multirow{2}{*}{ Grupos } & \multicolumn{3}{|c|}{ Estímulo dos Pais } & \multicolumn{3}{|c|}{ Motivação Geral } & \multicolumn{3}{|c|}{ Funcionamento Intelectual } \\
\hline & & $\mathrm{N}$ & Média $(d p)$ & $t$ & $\mathrm{~N}$ & Média $(d p)$ & $t$ & $\mathrm{~N}$ & Média $(d p)$ & $t$ \\
\hline \multirow{2}{*}{ Estudantes } & Piores & 10 & $\begin{array}{l}43,30 \\
(7,24)\end{array}$ & \multirow{2}{*}{$-3,33^{\star *}$} & 11 & $\begin{array}{l}44,18 \\
(8,45)\end{array}$ & \multirow{2}{*}{$-2,94^{\star *}$} & 12 & $\begin{array}{l}44,91 \\
(7,34)\end{array}$ & \multirow{2}{*}{$-2,70^{\star *}$} \\
\hline & Melhores & 104 & $\begin{array}{l}50,47 \\
(6,42)\end{array}$ & & 100 & $\begin{array}{l}50,44 \\
(6,50)\end{array}$ & & 93 & $\begin{array}{l}50,46 \\
(6,60)\end{array}$ & \\
\hline \multirow{2}{*}{ Pais } & Piores & 6 & $\begin{array}{l}33,50 \\
(9,91)\end{array}$ & \multirow{2}{*}{$-4,62^{\star \star}$} & 8 & $\begin{array}{l}32,75 \\
(8,50)\end{array}$ & \multirow{2}{*}{$-5,36^{\star \star}$} & 10 & $\begin{array}{l}36,60 \\
(9,90)\end{array}$ & \multirow{2}{*}{$-4,54^{\star *}$} \\
\hline & Melhores & 72 & $\begin{array}{l}51,05 \\
(8,87)\end{array}$ & & 68 & $\begin{array}{l}50,41 \\
(8,84)\end{array}$ & & 63 & $\begin{array}{l}50,87 \\
(9,13)\end{array}$ & \\
\hline \multirow{2}{*}{ Professores } & Piores & 10 & $\begin{array}{l}24,00 \\
(6,22)\end{array}$ & \multirow{2}{*}{$-11,21^{* *}$} & 11 & $\begin{array}{l}23,64 \\
(5,82)\end{array}$ & \multirow{2}{*}{$-12,63^{\star *}$} & 12 & $\begin{array}{l}23,92 \\
(6,61)\end{array}$ & \multirow{2}{*}{$-13,33^{* x}$} \\
\hline & Melhores & 104 & $\begin{array}{l}48,30 \\
(6,57)\end{array}$ & & 100 & $\begin{array}{l}48,38 \\
(6,20)\end{array}$ & & 93 & $\begin{array}{l}48,89 \\
(6,04)\end{array}$ & \\
\hline
\end{tabular}

${ }^{*} p<0,05{ }^{* *} p<0,00$

Observa-se, na Tabela 4, que os alunos com maior estímulo dos pais apresentaram escores significativamente mais elevados de habilidades sociais do que os alunos com pior estímulo dos pais. Este resultado foi o mesmo quando as habilidades sociais foram avaliadas 
pelos estudantes $(\mathrm{t}=-3,33 ; p=0,00)$, pelos pais $(\mathrm{t}=$ $4,62 ; p=0,00)$ e pelos professores $(\mathrm{t}=-11,21 ; p=0,00)$.

Motivação Geral para o Êxito Acadêmico: A Tabela 4 apresenta os resultados das habilidades sociais para os grupos com menor e maior motivação geral para o êxito acadêmico. Os alunos com maior motivação apresentaram escores significativamente mais elevados de habilidades sociais do que os alunos com pior motivação geral. Este resultado foi o mesmo quando as habilidades sociais foram avaliadas pelos estudantes $(\mathrm{t}=-2,94 ; p=0,00)$, pelos pais $(\mathrm{t}=-5,36$; $p=0,00)$ e pelos professores $(\mathrm{t}=-12,63 ; p=0,00)$.

Funcionamento Intelectual: Os escores de habilidades sociais para os grupos de alunos com melhor e pior funcionamento intelectual se encontram na Tabela 4. Os alunos com melhor funcionamento intelectual apresentaram escores significativamente mais elevados de habilidades sociais do que os alunos com pior funcionamento intelectual. Este resultado foi o mesmo quando as habilidades sociais foram avaliadas pelos estudantes $(\mathrm{t}=-2,70 ; p=0,00)$, pelos pais $(\mathrm{t}=$ $4,54 ; p=0,00)$ e pelos professores $(\mathrm{t}=-13,33 ; p=0,00)$.

\section{DISCUSSÃO}

A presente pesquisa identificou que $11,33 \%$ dos alunos avaliados apresentavam baixo desempenho acadêmico, sendo classificados como os "piores" alunos e que $7,82 \%$ apresentaram muita dificuldade de aprendizagem. Esses resultados representam um menor nível de dificuldades do que o encontrado por Carneiro e cols. (2003), ao avaliar o desempenho na escrita de crianças de escolas públicas da $3^{\mathrm{a}}$ série de Ensino Fundamental de Campinas $(15,2 \%)$. Essa inconsistência pode refletir diferenças reais no grau de dificuldade das crianças das duas amostras ou serem resultantes do tipo de avaliação realizada, pois uma avaliou a dificuldade geral de aprendizagem e a outra, especificamente a dificuldade na escrita.

A competência acadêmica dos alunos se mostrou significativamente relacionada com o seu nível socioeconômico, no presente estudo. Observam-se, nesses dados, semelhanças com os encontrados nos estudos de Carvalho (2004), de Barbosa (1999) e de Ferrão e cols. (2001), que também encontraram correlações positivas significativas entre estas duas variáveis. Como o tipo de escola freqüentado pelas crianças em nosso país depende do nível socioeconômico, os resultados obtidos quanto a esta variável foram os esperados, ou seja, crianças de escola particular apresenta- ram maior competência acadêmica do que as de escola pública, semelhante aos resultados obtidos por Dal Vesco e cols. (1998). No que se refere à influência do sexo, a melhor competência acadêmica das meninas confirma os dados da literatura (Barbosa, 1999; Carvalho, 2004; Souza e cols, 2003).

A competência acadêmica se mostrou significativamente relacionada com as habilidades sociais. Os resultados da presente pesquisa mostraram que quanto mais elaborado era o repertório de habilidades sociais das crianças, maior era sua competência acadêmica. Estes resultados estão de acordo com os estudos presentes na literatura, indicando que alunos com problemas de aprendizagem apresentavam maiores dificuldades em vários aspectos do funcionamento social (Estevanato e cols., 2003; Marturano e cols., 1993; Medeiros e cols., 2003).

Na presente pesquisa, observou-se que os escores de habilidades sociais foram mais elevados entre os alunos com alto desempenho acadêmico, comparativamente aos com baixo desempenho, o que foi também verificado por Lemos e Menezes (2002), utilizando o SSRS. Outra semelhança desses resultados pode ser encontrada nas pesquisas de Del Prette e Del Prette (2003), que compararam as habilidades sociais de alunos com e sem dificuldades de aprendizagem, observando que alunos sem dificuldades de aprendizagem apresentavam um repertório de habilidades sociais mais elaborado do que os com dificuldades de aprendizagem.

Estes resultados indicam a presença de uma relação, consistentemente observada na literatura, entre o repertório de habilidades sociais e o desempenho acadêmico das crianças, quando se comparou grupos de sujeitos e quando se correlacionou estas duas variáveis. Esta relação foi ainda confirmada em uma pesquisa onde se introduziu uma intervenção visando desenvolver as habilidades sociais, o que teve como efeito uma melhora significativa no desempenho acadêmico das crianças (Molina, 2003). Os resultados da presente pesquisa constituem, portanto, em uma nova confirmação para a relação entre estas duas variáveis, com dados de crianças de escolas de Ensino Fundamental de uma região brasileira ainda não estudada.

\section{CONCLUSÕES}

Embora a porcentagem de crianças com dificuldades de aprendizagem, encontrada na presente pesquisa, seja inferior à observada por Carneiro e cols. (2003), 
os resultados apontam para a necessidade destas crianças receberem uma atenção especial, pois as dificuldades de aprendizagem, além de resultar em vários problemas de ordem emocional e comportamental (Medeiros \& Loureiro, 2004), influenciam negativamente a vida futura dessas crianças (Marturano \& Loureiro, 2003). Além disso, esta porcentagem encontrada ainda é alta, demonstrando a necessidade de intervenções visando reduzir este número.

A presente pesquisa constatou que a competência acadêmica variou em função dos indicadores sociodemográficos e pôde identificar grupos com maiores dificuldades, como o grupo de meninos, o grupo de crianças de baixo nível socioeconômico e o de crianças de escolas públicas, mostrando que estes grupos se encontram em maior risco de desenvolver problemas escolares e, portanto, necessitam de uma atenção especial.

Adicionalmente, os resultados obtidos colocam em destaque a importância das habilidades sociais como um fator de proteção para o desempenho acadêmico das crianças e estão em concordância com a literatura da área. Tendo em vista as implicações negativas causadas pelo fracasso escolar no desenvolvimento posterior das crianças e sua estreita relação com o repertório de habilidades sociais, os dados encontrados apontam para a importância e a necessidade de programas de habilidades sociais tanto clínico como profilático a serem implementados nas escolas

\section{REFERÊNCIAS}

ABEP (2003). Critério de Classificação Econômica Brasil. Associação Brasileira de Empresas de Pesquisa. Obtido em 10 de fevereiro de 2006 do World Wide Web: www.abep.org/ codigosguias.

Bandeira, M., Rocha, S. S., Freitas, L. C., Del Prette, Z. A. P \& Del Prette, A. Habilidades sociais e variáveis sociodemográficas em estudantes do ensino fundamental. Psicologia em Estudo (no prelo).

Barbosa, M. L. O. (1999). Desempenho escolar e desigualdades sociais: Resultados preliminares de pesquisa. Trabalho apresentado no XXIII Encontro Anual da ANPOCS, Caxambu.

Bronfenbrenner, U. (1996). A ecologia do desenvolvimento humano: Experimentos naturais e planejados. Porto Alegre: Artes Médicas.

Caballo, V. E. (2003). Manual de avaliação e treinamento das habilidades sociais. São Paulo: Livraria Santos Editora.

Carneiro, G. R. S., Martinelli, S. C. \& Sisto, F. F. (2003). Autoconceito e dificuldades de aprendizagem na escrita. Psicologia: Reflexão e Critica, 16(3), 427-434.

Carvalho, M. P. (2003). Sucesso e fracasso escolar: Uma questão de gênero. Educação e Pesquisa, 29(1), 185-193.
Carvalho, M. P. (2004). Who are the boys failing at school? Cadernos de Pesquisa, 34(121), 11-40.

Dal Vesco, A., Mattos, D., Beninca, C. \& Ciomara (1998). Correlação entre WISC e rendimento escolar na escola pública e na escola particular. Psicologia: Reflexão e Crítica, 11(3), 481-495.

Del Prette, A. \& Del Prette, Z. A. P. (1995). Interações sociais em sala de aula: Representações do professor. Em XVII International School Psychology (Org.), Anais do II Congresso Nacional de Psicologia Escolar (pp. 426-430). Campinas: Átomo.

De Prette, A. \& Del Prette, Z. A. P. (2001). Psicologia das relações interpessoais: Vivências para o trabalho em grupo. Petrópolis: Vozes.

Del Prette, Z. A. P. (2003). Prevenção de dificuldades de aprendizagem e da violência: desenvolvimento e tecnologia para a avaliação e promoção de habilidades sociais. Relatório de auxílio à pesquisa no exterior (pós-doutorado) aprovado pela FAPESP, Processo ${ }^{\circ}$ 02-00105-2). São Paulo (SP).

Del Prette, Z. A. P. \& Del Prette, A. (1998) Desenvolvimento interpessoal e educação escolar: $\mathrm{O}$ enfoque das habilidades sociais. Temas em Psicologia, 6(3), 205-215.

Del Prette, Z. A. P. \& Del Prette, A. (1999). Psicologia das habilidades sociais: Terapia e educação. Petrópolis: Vozes.

Del Prette, Z. A. P. \& Del Prette, A. (2002). Avaliação de habilidades sociais de crianças com um inventário multimídia: Indicadores sociodemográficos associados à freqüência versus dificuldade. Psicologia em Estudo, 7(1), 61-73.

Del Prette, Z. A. P. \& Del Prette, A. (2003). Habilidades sociais e dificuldades de aprendizagem: Teoria e pesquisa sob um enfoque multimodal. Em Habilidades sociais, desenvolvimento e aprendizagem: Questões conceituais, avaliação e intervenção (p. 167-206). Campinas: Alínea.

Del Prette, Z. A. P. \& Del Prette, A. (2005). Psicologia das habilidades sociais na infância: Teoria e prática. Petrópolis: Vozes.

Del Prette, Z. A. P., Del Prette, A. \& Barreto, M. C. M. (1999). Análise do Inventário de Habilidades Sociais (IHS) em uma amostra de universitários. Psicologia: Teoria e Pesquisa, 14(3), 119-128.

Del Prette, Z. A. P., Del Prette, A., Pontes, A. C. \& Torres, A. C. (1998). Efeitos de um programa de intervenção sobre aspectos topográficos das habilidades sociais de professores. Psicologia Escolar e Educacional, 2, 11-22.

Erickson, E. H. (1976). Infância e sociedade. Rio de Janeiro: Zahar.

Estevanato, I. S., Loureiro, S. R., Linhares, M. B. M. \& Marturano, E. M. (2003). Autoconceito de crianças com dificuldades de aprendizagem e problemas de comportamento. Psicologia em Estudo, 8(1), 67-76.

Ferrão, M. E., Beltrão, K. I. \& Santos, D. P. (2001). Política de não-repetência e a qualidade da educação: Evidências obtidas na modelagem dos dados da $4^{a}$ série do Saeb-99. Brasília: MEC/Inep.

Ferreira, M. \& Marturano, E. (2002). Ambiente familiar e os problemas de comportamento apresentados por crianças com baixo desempenho escolar. Psicologia: Reflexão e Crítica, 15(1), 1-11. 
Freller, C. C. (1999). Pensando com Winnicott sobre alguns aspectos relevantes ao processo de ensino e aprendizagem. Psicologia USP, 10(2), 189-203.

Greshan, F. M. \& Elliot, S. N. (1990). Social Skills Rating System: Manual. Circle Pines, MN: American Guidance Service.

Lemos, M. S. \& Meneses, H. I. (2002). A avaliação da competência social: Versão portuguesa da forma para professores do SSRS. Psicologia: Teoria e Pesquisa, 18(3), 267-274.

Lindahl, N. Z. (1998). Personalidade humana e cultura: Aplicações educacionais da teoria de Erik Erikson. Revista Brasileira de Estudos Pedagógicos, 69(163), 492-509.

Marinho, M. (2003). Comportamento anti-social infantil: Questões teóricas e de pesquisa. Em Z. A. P. Del Prette \& A. Del Prette (Orgs.), Habilidades sociais, desenvolvimento e aprendizagem (p. 61-81). Campinas: Alínea.

Marturano, E. M. (1997). A criança, o insucesso escolar precoce e a família: Condições de resiliência e vulnerabilidade. Em E. M. Marturano, S. R. Loureiro \& A. W. Zuardi (Orgs.), Estudos em saúde mental (p. 132-145). Ribeirão Preto: Comissão de PósGraduação em Saúde Mental da FMRP/USP.

Marturano, E. M., Linhares, M. B. M. \& Parreira, V. L. C. (1993). Problemas emocionais e comportamentais associados a dificuldades na aprendizagem escolar. Medicina, 26, 161-175.

Marturano, E. M. \& Loureiro, S. R. (2003). O desenvolvimento socioemocional e as queixas escolares em habilidades sociais. Em A. Del Prette \& Z. A. P. Del Prette (Orgs.), Desenvolvimento e aprendizagem: Questões conceituais, avaliação e intervenção (p. 259-291). Campinas: Alínea.

Medeiros, P. C. \& Loureiro, S. R. (2004). Observação clínica do comportamento de crianças com queixa de dificuldade de aprendizagem. Em E. M. Marturano, M. B. M. Linhares \& S. R.
Loureiro (Orgs.), Vulnerabilidade e proteção: Indicadores na trajetória de desenvolvimento escolar (p. 107-122). São Paulo: Casa do Psicólogo.

Medeiros, P. C., Loureiro, S. R., Linhares, M. B. M. \& Marturano, E. M. (2000). A auto-eficácia e aspectos comportamentais em crianças com dificuldades de aprendizagem. Psicologia: Reflexão e Critica, 13(3), 327-336.

Medeiros, P. C., Loureiro, S. R., Linhares, M. B. M. \& Marturano, E. M. (2003). O senso de auto-eficácia e o comportamento orientado para aprendizagem em crianças com queixa de dificuldade de aprendizagem. Estudos de Psicologia (Natal), 8(1), 93-105.

Molina, R. C. (2003). Habilidades sociais e dificuldades de aprendizagem: Explorando relações funcionais. Dissertação de Mestrado. Programa de Pós-Graduação em Educação Especial. Universidade Federal de São Carlos.

Patto, M. H. S. (1987). A produção do fracasso escolar: Histórias de submissão e rebeldia (v. 1-2). Tese de Livre-Docência, Instituto de Psicologia da Universidade de São Paulo, São Paulo.

Severson, H. H. \& Walker, H. M. (2002). Proactive approaches for identifying children at risk for sociobehavioral problems. Em K. L. Lane, F. M. Gresham \& T. E. O’Shaughnessy (Orgs.), Children whith or at risk for emotional and behavioral disorders (p. 33-53). Boston: Allyn \& Bacon.

Souza, M. P. R., Teixeira, D. C. S. \& Silva, M. C. Y. G. (2003). Conselho Tutelar: Um novo instrumento social contra o fracasso escolar? Psicologia em Estudo, 8(2), 71-82.

Recebido: 02/06/2005 Revisado: $13 / 03 / 2006$ Aceito: $20 / 03 / 2006$

\section{Nota:}

${ }^{1}$ Este artigo apresenta parte dos resultados associados ao Projeto Temático de Pesquisa: Habilidades sociais em crianças: Desenvolvendo instrumentos de avaliação e investigando variáveis relacionadas, coordenado pela Prof ${ }^{\mathrm{a}}$. Dr ${ }^{\mathrm{a}}$. Zilda A. P. Del Prette. Projeto Temático de Pesquisa apoiado pelo CNPq (Processo 504660/2003-9) e aprovado pelo Comitê de Ética da UFSCar (Protocolo 002/04, de 26/03/2004).

\section{Sobre os autores:}

Marina Bandeira: Professora Adjunta da Universidade Federal de São João del Rei (UFSJ). Pesquisadora-bolsista do CNPq. Doutora Ph.D. pela Université de Montréal. Pós-doutora pela McGill University e Université de Montreal.

Sandra Silva Rocha: Professora Assistente da Universidade Federal de São João del Rei (UFSJ). Mestre pela Universidade Federal do Rio de Janeiro (UFRJ).

Luisa Gonçalves Pires: aluna do curso de graduação de Psicologia, participante do Programa Institucional de Iniciação Científica (PIIC) da UFSJ.

Zilda Aparecida Pereira Del Prette: Professora Titular da Universidade Federal de São Carlos (UFSCar). Pesquisadora-bolsista do CNPq. Doutora pela Universidade de São Paulo (USP), Pós-doutora pela Universidade da Califórnia - Riverside.

Almir Del Prette: Professor Titular da Universidade Federal de São Carlos (UFSCar). Pesquisador-bolsista do CNPq. Doutor pela Universidade de São Paulo (USP).

Endereço para correspondência: Marina Bandeira - Rua Antonia da Encarnação Xavier, 120 - 36325-000 - Tiradentes/MG Endereço eletrônico: marina@mgconecta.com.br. 